\title{
Protein phosphatase 2C-alpha knockdown reduces angiotensin II-mediated skeletal muscle wasting via restoration of mitochondrial recycling and function
}

Alexander Michael Tabony ${ }^{1}$, Tadashi Yoshida', Sergiy Sukhanov ${ }^{1}$ and Patrice Delafontaine $e^{1,2^{*}}$

\begin{abstract}
Background: Circulating angiotensin II (Angll) is elevated in congestive heart failure (CHF), and leads to skeletal muscle wasting, which is strongly associated with poor patient outcomes. We previously found that Angll upregulates protein phosphatase 2C-alpha (PP2Ca) and dephosphorylates AMP-activated protein kinase (AMPK), a critical regulator of cellular metabolism, in skeletal muscle.

Methods: To determine the role of PP2Ca in Angll-induced wasting, gastrocnemius (Gas) muscles of FVB mice were injected with scrambled or PP2Ca siRNA and mice were infused with saline or Angll for 4 days.

Results: Knockdown of PP2Ca reduced Angll wasting, blocked Angll upregulation of PP2Ca, increased p-T172-AMPK, and inhibited Angll-mediated reductions in peroxisome proliferator-activated receptor- $\gamma$ coactivator-1a (PGC-1a), nuclear respiratory factor 1 (NRF1), mitochondrial transcription factor A (TFAM), in complex IV activity, and in ATP levels. Angll impaired the rate of autophagy as determined by a 2.4-fold increase in p62/SQSTM1 (p62) accumulation. This induction was reduced by PP2Ca knockdown, which also increased beclin-1 expression and microtubule-associated protein 1 light chain 3 (LC3)-II conversion in Angll-infused Gas. Angll reduced activating S555 phosphorylation of UNC-51-like kinase 1 (ULK1), a critical regulator of autophagosome formation, and increased inhibitory S757 ULK1 phosphorylation and these effects were prevented by PP2Ca siRNA.

Conclusions: Angll inhibited AMPK activity and reduced PGC-1a and TFAM expression (thereby inhibiting mitochondrial biogenesis) and impaired ULK1 activation and autophagy (thereby also inhibiting clearance of damaged mitochondria), resulting in mitochondrial dysfunction, decreased ATP, and wasting. Knockdown of PP2Ca normalized AMPK activity, PGC-1a, NRF1, and TFAM levels and blocked Angll inhibition of ULK1, leading to improved mitochondrial biogenesis/ recycling/function, energy production, and inhibition of Angll-induced wasting. These results demonstrate novel effects of Angll on cellular metabolism that are likely critical in mediating the muscle wasting that is a hallmark of CHF.
\end{abstract}

Keywords: Angiotensin II, PP2C, Muscle atrophy, Mitochondria, Autophagy

\footnotetext{
* Correspondence: pdelafon@tulane.edu

'Tulane University Department of Medicine, Heart and Vascular Institute, New Orleans, LA 70112, USA

${ }^{2}$ Heart and Vascular Institute, Tulane University School of Medicine, 1430

Tulane Ave. SL-48, New Orleans, LA, USA
} 


\section{Background}

In conditions such as congestive heart failure and chronic kidney disease, where loss of lean body mass correlates strongly with poor prognosis [1-3], circulating angiotensin II (AngII) is elevated, and is a likely major contributor to skeletal muscle wasting associated with these diseases. Indeed, we have shown that AngII infusion in rodents induces muscle atrophy which is related to multiple mechanisms including activation of the ubiquitin proteasome pathway (UPP) [4,5], inhibition of the insulin/IGF-1/Akt/ mammalian target of rapamycin (mTOR) axis [5-13], reduction in appetite [14-16], and activation of apoptosis $[10,17]$. The effects of AngII include indirect effects via circulating mediators $[6,13,18-22]$ and direct effects to reduce regeneration $[16,23]$.

While it has been well established that AngII causes skeletal muscle atrophy, much less is known about the metabolic and bioenergetic effects of AngII, or how these effects relate to AngII wasting. Protein breakdown in skeletal muscle is predominantly mediated by the UPP and the autophagy-lysosome system, but the specific role of autophagy in AngII skeletal muscle wasting is not known. We originally reported that AngII impaired AMP-activated protein kinase (AMPK) activation in muscle, likely via upregulation of protein phosphatase 2C-alpha (PP2C $\alpha$ ) (AngII did not inhibit kinases upstream to AMPK), leading to mitochondrial dysfunction and ATP depletion, and that direct activation of AMPK with 5-aminoimidazole-4-carboxamide ribonucleoside (AICAR) prevented AngII wasting $[14,16]$. The aims of this study were to determine the mechanistic link between PP2C $\alpha$ and AngII wasting and to uncover the mechanisms involved in AngII-induced mitochondrial dysfunction and ATP depletion in skeletal muscle.

\section{Methods}

\section{In vivo muscle siRNA electroporation}

Four separate siRNAs targeted to PP2C $\alpha$ mRNA (SureSilencing $^{\text {mix }}$ siRNA set, Qiagen/SA Biosciences) were tested for knockdown efficiency in vivo, by injecting gastrocnemius (Gas) muscles of ketamine/xylazine anesthetized FVB mice (male, 8 to 10 weeks old, Charles River) with $2.5 \mu \mathrm{g}[24,25]$ of each siRNA and control scrambled siRNA in sterile saline at 5 sites using $50 \mu \mathrm{L}$ Hamilton syringes with 22 gauge needles, followed by immediate electroporation of the muscle using a BTX Harvard Apparatus ECM 830 Electro Square Porator ${ }^{\mathrm{rt}}$ with the following settings: $50 \mathrm{~V}$ [24,25], $20 \mathrm{~ms}$ pulse duration, $0.5 \mathrm{~cm}$ between electrodes, 8 unipolar pulses [25,26], and $1 \mathrm{~s}$ between pulses. The target sequences of the four PP2C $\alpha$ siRNAs were as follows: siRNA A - CCAAGATATTTCT GAGACATT, siRNA B - CCAGATACAAATTACCT GTTT, siRNA C - CTTGGTGGATGGGCAGATCTT, and siRNA D - CAAGCTGCAATCATGGAACTT. After determining the most effective siRNA, it was used for time course $(1,3,5,7$, and 11 days) and dose response experiments (vehicle, $2.5 \mu \mathrm{g}, 5 \mu \mathrm{g}, 7.5 \mu \mathrm{g}, 10 \mu \mathrm{g}, 12.5 \mu \mathrm{g}$ siRNA) to further optimize target knockdown in Gas in vivo.

\section{Experimental design}

In the experimental setting, $5 \mu \mathrm{g}$ of PP2C $\alpha$ siRNA and scrambled siRNA were electroporated into contralateral Gas muscles (alternating right and left between animals). Three days later, mice were implanted subcutaneously with osmotic minipumps (Alzet 1007D) continuously infusing either sterile saline (control) or $1,000 \mathrm{ng} / \mathrm{kg} / \mathrm{min}$ AngII (Phoenix Pharmaceuticals). This dose of AngII yields a 2.8-fold increase in plasma AngII that is within the pathophysiological ranges observed in patients with congestive heart failure (CHF) and chronic kidney disease [12,14,27-30]. Mice were sacrificed after another 4 days, muscles were collected, weighed, and either used fresh or embedded in Allprotect Tissue Reagent (Qiagen) and stored at $-80^{\circ} \mathrm{C}$ until processing. Cryosections were prepared by incubating Gas muscles in $50 \%$ optimal cutting temperature (OCT) compound (Tissue-Tek, USA) for 15 min on ice followed by freezing in 100\% OCT. Eight micron serial cross sections were prepared from the middle of each muscle and kept at $-80^{\circ} \mathrm{C}$ until processing. The animal protocols were approved by Tulane University Institutional Animal Care and Use Committee.

\section{Biochemical assays}

Frozen tissue was blotted dry of excess Allprotect reagent and pulverized with a mortar and pestle over liquid nitrogen. Aliquots of each pulverized muscle were prepared as previously described [14] for mitochondrial quantification (via DNA isolation followed by real-time PCR using primers specific for mitochondrial DNA and genomic DNA), complex IV activity (Mitochondria Activity Assay Kit, BioChain), ATP quantification (ATP determination kit, Invitrogen), quantitative real-time RT-PCR (using Qiagen/ SuperArray optimized primers for PP2C $\alpha$, p62/SQSTM1, LC3A, Fbxo32, and Trim63, plus $\beta$-actin and HPRT1 housekeeping genes), and SDS-PAGE/western blotting. Primary antibodies utilized were as follows: from Abcam: PPM1A (ab14824), PP2C alpha + PP2C beta (ab27267), PGC-1 $\alpha$ (ab54481), Fbxo32 (ab74023), MuRF1 (ab77577), Mitofusin-1, TTC11 (Fis1, ab71498), MARCH5 (ab77585), UCP3 (ab3477), Mitofusin-2 (ab56889), TFAM (ab131607), OPA1 (ab55772), Mff (ab81127), and MitoProfile Total OXPHOS Rodent WB Antibody Cocktail (Abcam/MitoSciences, ab110413), from Cell Signaling Technology: p-T172-AMPK $\alpha$ (2531), AMPK $\alpha$ (2532), p-S473Akt (4058), p-T308-Akt (5106), Akt (9272), Phospho-Fox01 (Thr24)/Fox03a (Thr32) Antibody (9464), p-S413-Fox03a (8174), Fox01 (2880), Fox03a (2497), caspase-3 (9662), cleaved caspase-3 (9664), TCF11/NRF1 (8052), p-S616- 
DRP1 (3455), DRP1 (5391), Ubiquitin (3936), Beclin-1 (3495), LC3A/B (12741), LAMP1 (9091), p-S757-Ulk1 (6888), p-S555-Ulk1 (5869), Ulk1 (8054), SQSTM1/p62 (5114), $\beta$-Actin, and $\alpha$-tubulin (2144), from Enzo: Rpt6 (BML-PW9265).

\section{Immunohistochemistry}

Serial skeletal muscle frozen cross sections were stained for LC3 and p62 using rabbit a/LC3 pAb from MBL Co., Ltd (Cat\# PM036, Japan) or mouse a/SQSTM1/p62 mAb from Abcam (cat\# ab56416, USA), respectively. Sections were fixed with $4 \%$ paraformaldehyde (15 min), treated with $0.1 \%$ Triton $\mathrm{X}-100$ in PBS, and stained with primary $\mathrm{a} / \mathrm{b}$ for $1 \mathrm{~h}$ at room temperature. Biotin-conjugated anti-mouse (or anti-rabbit) IgG (1:400, Vector Labs, cat\# BA2000 and BA1000, respectively) were applied for sections for $45 \mathrm{~min}$ followed by incubation with streptavidin-Alexa594 conjugate (1:500) plus DAPI (both from Invitrogen). Antibody specificity was verified routinely by staining of serial sections with 'normal' IgG (obtained from an unimmunized animal of the same species as primary antibody, Santa Cruz Biotechnology, Santa Cruz, CA, USA). We did not observe any detectable immunopositivity on 'normal' IgG-stained sections (data not shown).

\section{Statistical analysis}

All data are presented as means +/- standard errors (SEM, $\mathrm{n}$ indicated in figure legends and within each individual group/bar). GraphPad software (Version 6.03 for Windows, GraphPad Software, San Diego, CA, USA) was used to perform the statistical analysis. Differences between groups were determined by two-way ANOVA followed by Holm-Sidak's multiple comparisons test (for experiments involving both AngII infusion and siRNA intervention, as well as for time courses), or by one-way ANOVA followed by Tukey post-tests (determination of optimal siRNA target sequence and dose responses with a single siRNA) as appropriate. A value of $P<0.05$ was considered statistically significant.

\section{Results}

\section{Knockdown of PP2Ca in vivo}

Four days after electroporation, PP2C $\alpha$ siRNAs B and C both significantly reduced expression of PP2C $\alpha$ mRNA in Gas by approximately $37 \%(P<0.05)$, while siRNA A proved to be the most effective with a $57 \%$ reduction $(P<0.001)$ in mRNA compared to control, and siRNA D failed to reduce expression of PP2C $\alpha$ (Figure 1A). A total of $2.5 \mu \mathrm{g}$ of PP2C $\alpha$ siRNA A significantly reduced gene expression from 1 to 7 days post electroporation, with a peak suppression from baseline of $75 \%$ measured at day 3 , and returning to normal by day 11 (Figure 1B). There was a non-statistically significant trend for transient suppression of PP2C $\alpha$ mRNA expression with scrambled siRNA/electroporation, which returned to normal between days 3 and 5 (Figure 1B). Similarly, PP2C $\alpha$ protein tended to be reduced by PP2C $\alpha$ siRNA A throughout the time course, with a significant reduction of $58 \%$ at day 7 (Figure $1 \mathrm{C}$ ), while AMPK phosphorylation was significantly increased at days 1,5 , and 7 (Figure 1D). The dose response performed at day 7 indicated that $5 \mu \mathrm{g}$ of siRNA was the optimal dose, with a $51 \%$ reduction in expression compared to $31 \%$ with $2.5 \mu \mathrm{g}$, while scrambled siRNA did not reduce expression at all (Figure 1E). PP2C $\alpha$ protein expression was significantly reduced at all doses of $\mathrm{PP} 2 \mathrm{C} \alpha$ siRNA (Figure $1 \mathrm{~F}$ ), and there was a concomitant 2.3-fold increase in AMPK phosphorylation with $5 \mu \mathrm{g}$ of PP2C $\alpha$ siRNA (Figure $1 G$ ).

\section{PP2Ca knockdown prevented Angll skeletal muscle wasting}

AngII-infused mice lost $12.5 \%$ of total body mass $(P<0.001)$ and $26 \%$ of Gas mass $(P<0.001)$ compared to saline-infused controls at day 4 (Figure $2 \mathrm{~A}$ and $\mathrm{B}$ ). Two unique PP2C $\alpha$ siRNAs significantly rescued the AngIIinduced reduction in Gas mass by approximately 37\% $(P<0.001$ and $P<0.05$ respectively, Figure $2 \mathrm{~B})$. As an additional control, we measured weights of ipsilateral quadriceps muscles, which were not treated with siRNA. As expected, there was no rescue in the quadriceps of the legs receiving either PP2C $\alpha$ siRNA in Gas (Figure 2C). AngII also reduced average cross sectional area of individual Gas muscle fibers (Figure 2D) and induced a frequency shift towards smaller fibers (Figure 2E), and PP2Ca siRNA prevented the AngII-induced reductions in fiber size (Figure 2D and E).

\section{PP2Ca knockdown restored AMPK activation and PGC-1a expression to activate mitochondrial biogenesis}

Infusion of AngII caused a 46\% increase in PP2C $\alpha$ protein expression $(P<0.0001)$, consistent with our previous findings [14], and two separate PP2C $\alpha$ siRNAs significantly reduced basal PP2C $\alpha$ expression and blocked the AngIImediated increase (down to a $13 \%$ increase, $P<0.001$ and $P<0.05$ respectively, Figure $3 \mathrm{~B})$. Consistent with increased PP2C $\alpha$ expression, AngII reduced activating T172 AMPK phosphorylation by $37 \%(P<0.05$, Figure $3 C)$, and PP2C $\alpha$ knockdown with two unique siRNAs increased AMPK phosphorylation by 2-fold and 1.8-fold respectively compared to scrambled siRNA (Figure 3C). Active AMPK increases expression of PGC-1 $\alpha$, which is a critical mediator of mitochondrial biogenesis, increasing the expression of transcription factors NRF1 and TFAM, which coordinate expression of mitochondrial genes and are critical for transcription of mitochondrial DNA (mtDNA) [31-33]. PGC- $1 \alpha$ expression was reduced by $29 \%$ with infusion of AngII $(P<0.001$, Figure 3D), with a near complete rescue by PP2C $\alpha$ knockdown using two separate siRNAs $(P<0.05$, Figure 3D). Similarly, AngII also tended to 


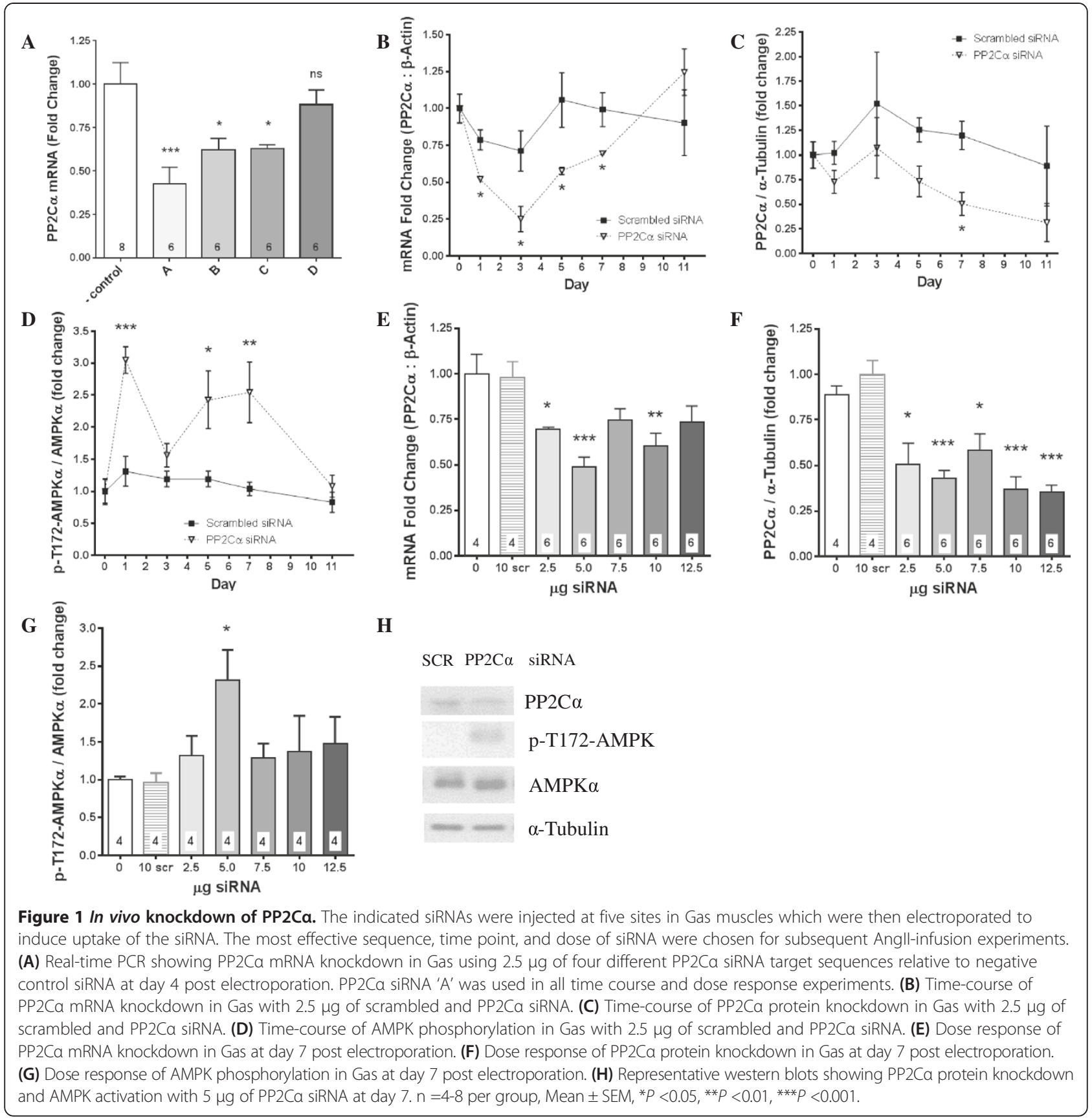

reduce expression of NRF1 (Figure 3E), and significantly reduced TFAM (Figure 3F), while PP2C $\alpha$ knockdown significantly increased expression of both NRF1 (Figure 3E) and TFAM (Figure 3F). Together, these results indicate that knockdown of PP2Ca prevented the AngII-mediated suppression of mitochondrial biogenesis.

\section{PP2Ca siRNA-mediated rescue of Angll wasting is} independent of the Akt-FOXO signaling axis

Although AngII greatly reduced activating S473 and T308 Akt phosphorylation (by $87 \%$ and $67 \%$, respectively,
$P<0.001$, Figure $4 \mathrm{~A}$ and $\mathrm{B}$ ), as well as downstream Akt-mediated FoxO1/FoxO3a phosphorylation (by 61\%, $P<0.001$, Figure 4C), PP2C $\alpha$ knockdown did not reverse those reductions, and had no effect on basal Akt and FOXO phosphorylation. Consistent with Akt and FOXO phosphorylation status, AngII also significantly increased gene expression of the E3 ubiquitin ligases atrogin-1 (Figure 4E), and MuRF1 (Figure 4F), while also increasing expression of atrogin-1 protein (Figure 4G), but not MURF1 protein (Figure 4H). PP2C $\alpha$ knockdown did not significantly reduce AngII-mediated induction of E3 ligase 


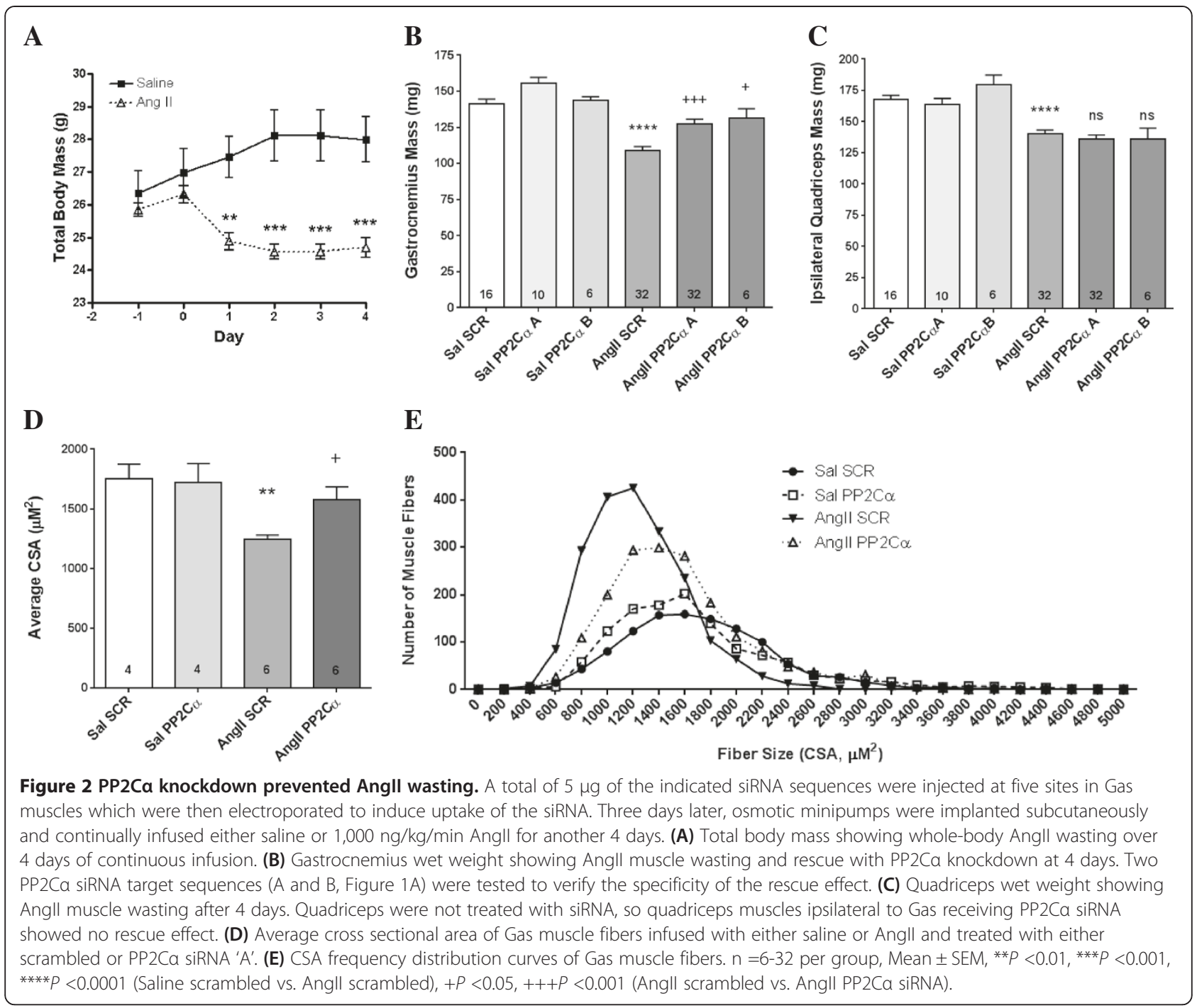

expression, and had no effect on basal expression of these two E3 ligases (Figure 4E-H). Further, phosphorylation at S413-FOXO, which is mediated by AMPK was not altered with AngII or PP2Ca knockdown (Figure 4D). Since knockdown of $\mathrm{PP} 2 \mathrm{C} \alpha$ did not prevent Akt inhibition, FOXO activation, and increased expression of atrogin- 1 or MURF1 by AngII, other pathways must be involved in the ability of PP2C $\alpha$ knockdown to reduce AngII-induced wasting.

PP2Ca knockdown increased mitochondrial content and ameliorated Angll-induced mitochondrial dysfunction

We have previously shown that AngII reduced skeletal muscle ATP levels, which could be prevented by activating AMPK with AICAR [14]. Therefore, we analyzed parameters of mitochondrial content and function with PP2C $\alpha$ knockdown. There was no observed change in the expression of uncoupling protein-3 (UCP3), or of electron transport chain complexes I-IV with AngII or
PP2Ca knockdown (Figure 5A-E), but AngII reduced ATP-synthase (complex V) expression by $68 \%(P<0.0001$, Figure 5G), and PP2C $\alpha$ siRNA did not restore complex V expression to normal (Figure 5F). Although AngII did not alter mitochondrial copy number compared to control, knockdown of PP2Ca increased basal mitochondrial content by $98 \%$ and by $46 \%$ in AngII infused Gas compared to scrambled siRNA, consistent with the trends observed in expression of PGC- $1 \alpha(P<0.05$, Figure $5 G)$.

Expression of cytochrome $C$ oxidase (complex IV) was not reduced (if anything there was a trend for increased expression with AngII, Figure 5E), but complex IV activity was reduced by $70 \%$ with AngII $(P<0.0001)$, and PP2C $\alpha$ knockdown partially blocked the effect of AngII such that complex IV activity was reduced by only $48 \%(P<0.05$, Figure $5 \mathrm{H})$. Quantification of ATP in Gas revealed a parallel trend, with a $74.5 \%$ reduction with AngII $(P<0.0001)$, which was partially inhibited to a $49 \%$ reduction by PP2C $\alpha$ knockdown $(P<0.05$, Figure $5 \mathrm{I})$. PP2C $\alpha$ knockdown did 


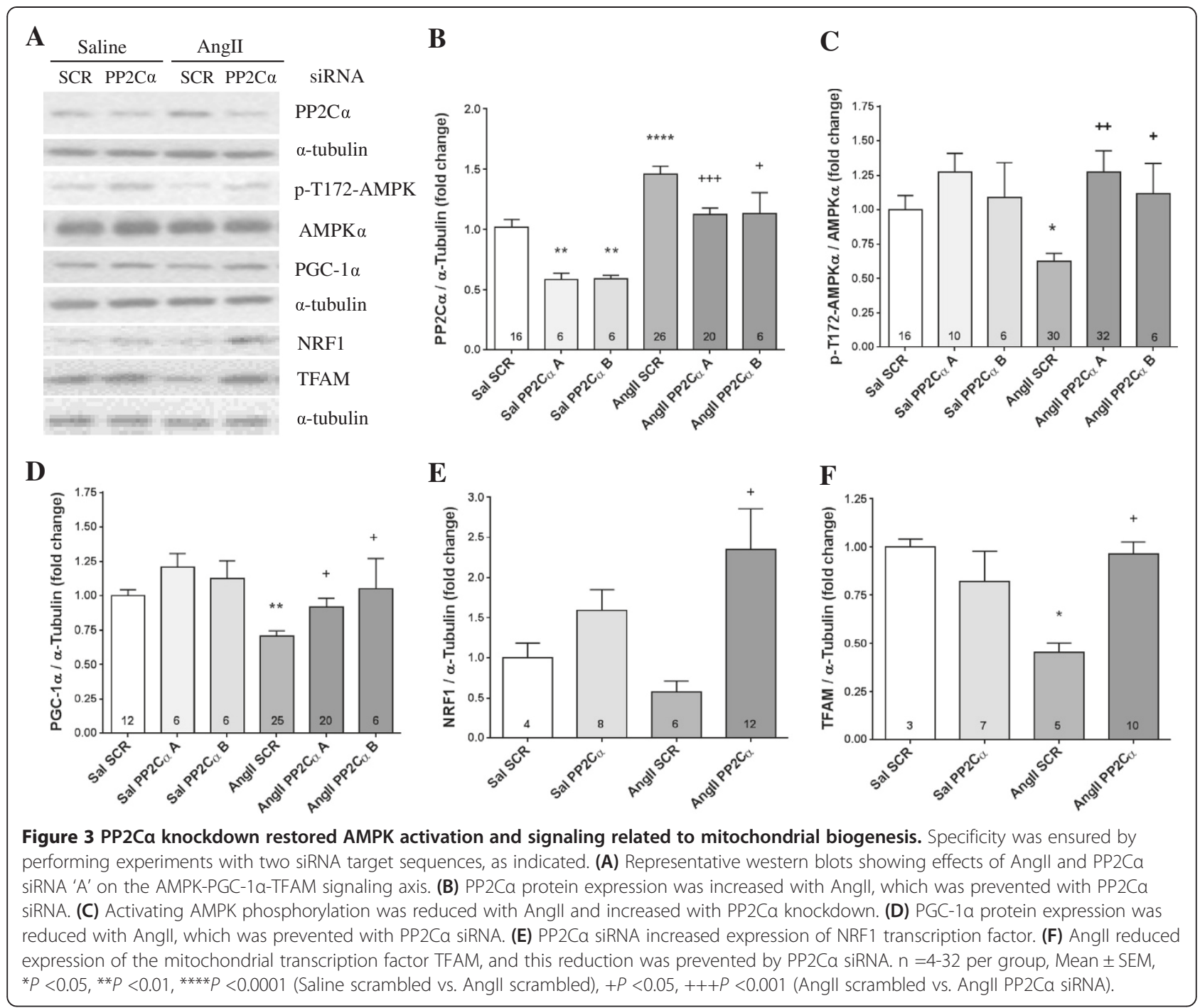

not alter complex IV activity or ATP content in the absence of AngII.

Prolonged mitochondrial dysfunction and bioenergetic stress can lead to initiation of apoptosis, which is characterized by caspase- 3 release and activation [34,35], and activation of caspase-3 is known to be required for AngII wasting [4,17]. Expression of total caspase-3 did not change with AngII or PP2C $\alpha$ knockdown, but $17 \mathrm{KDa}$ cleaved activated caspase-3 was increased 3.4-fold with AngII $(P<0.0001)$ and PP2C $\alpha$ siRNA reduced that increase to only 1.6 -fold, while not changing basal caspase activation $(P<0.001$, Figure $6 \mathrm{~A})$. Because caspase-3 activation has been linked to proteasome activation via cleavage of the 19S regulatory cap ATP-ase Rpt6 [17], we also assessed Rpt6 cleavage with AngII and PP2C $\alpha$ knockdown. AngII increased Rpt6 cleavage by 2 -fold without altering total Rpt6 expression $(P<0.05)$, and that increased cleavage was blunted by PP2C $\alpha$ siRNA $(P<0.05$, Figure 6B).
PP2C $\alpha$ knockdown in saline controls had no effect on Rpt6 cleavage. Ubiquitin-conjugated proteins were also increased with AngII, indicating an increase in substrates for the proteasome, while this increase tended to be reduced with PP2C $\alpha$ knockdown but did not approach significance (Figure 6C).

Collectively, these data indicate that AngII caused profound mitochondrial dysfunction without significantly altering total mitochondrial load, while also implicating activation of the proteasome. Mitochondrial copy number is coordinated simultaneously by mitochondrial biogenesis and mitochondrial turnover via mitophagy. As such, the fact that AngII did not alter mitochondrial content compared to control while significantly inhibiting pathways critical to mitochondrial biogenesis implicates an effect of AngII on autophagy/mitophagy. AngII-induced mitochondrial dysfunction was associated with activation of apoptotic signaling as evidenced by caspase- 3 activation. 


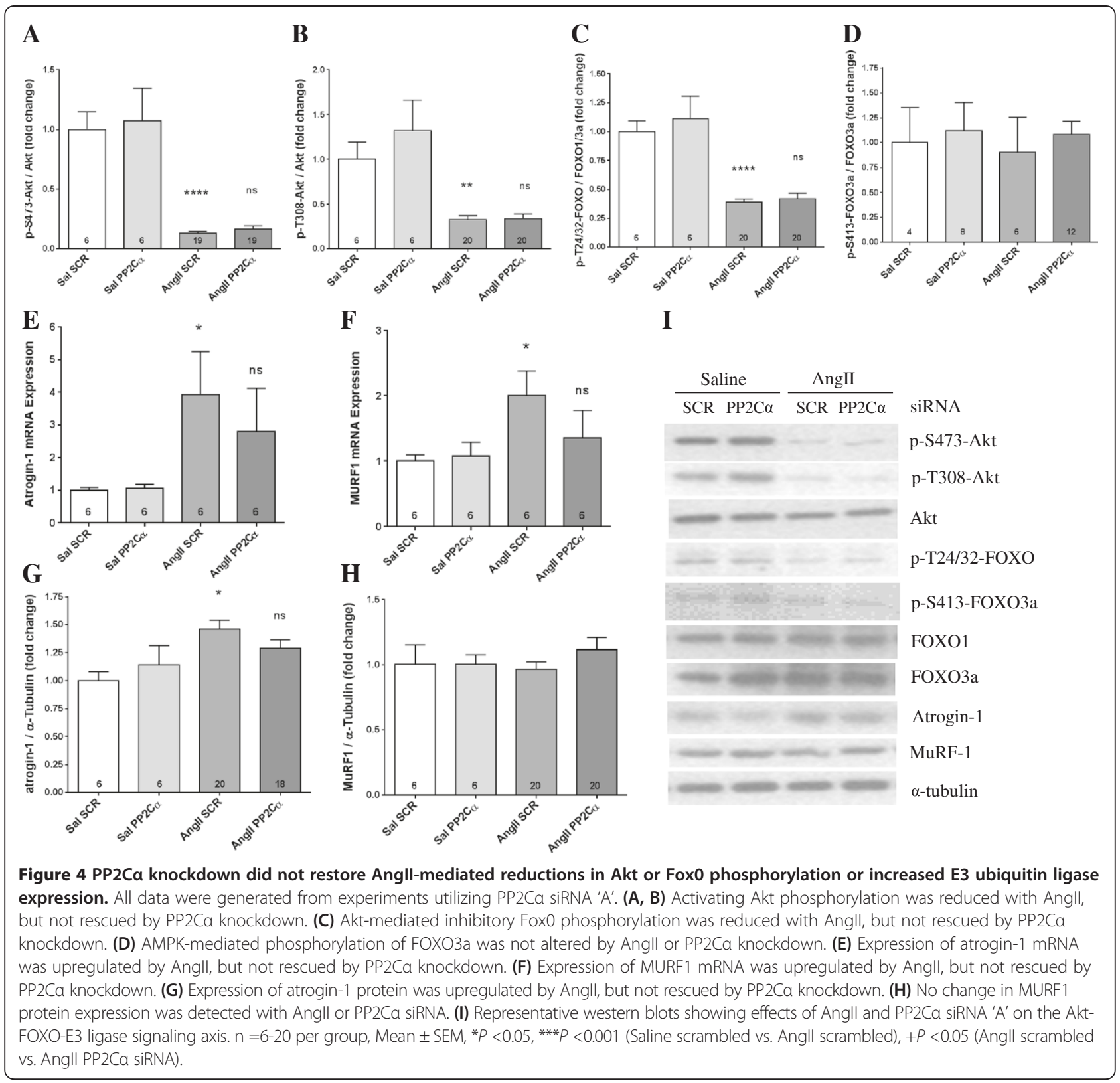

Further, PP2Ca knockdown partially prevented AngIIinduced mitochondrial dysfunction, caspase-3 activation, and Rpt6 cleavage.

\section{PP2Ca siRNA prevented Angll-mediated inhibition of autophagy}

Healthy mitochondrial function is preserved by balancing mitochondrial biogenesis and recycling via mitophagy. Since AngII reduced PGC- $1 \alpha$ without reducing total mitochondrial load in Gas, this indicated a potential impairment in mitophagy. To evaluate the role of autophagy in AngII-mediated mitochondrial dysfunction and wasting, we quantified several autophagy markers, including p62 accumulation, beclin-1 expression, and LC3-II conversion.
Expression of $\mathrm{p} 62$ protein was increased by 2.4-fold with AngII $(P<0.0001)$, and PP2C $\alpha$ siRNA blunted that increase to 1.5 -fold, while not altering basal p62 $(P<0.05$, Figure 7A and $\mathrm{B}$ ), and neither AngII nor PP2C $\alpha$ siRNA had any effect on transcription of p62 (Figure 7H), indicating an inhibition of autophagic flux with AngII and prevention of that inhibition with knockdown of PP2Ca. This is corroborated by the 2.8-fold increase in LC3-II conversion with PP2C $\alpha$ knockdown over AngII alone, indicative of increased mature autophagosome formation with PP2C $\alpha$ knockdown $(P<0.05$, Figure $7 \mathrm{~A}$ and $\mathrm{C})$. LC3A mRNA expression (Figure 7I) was not significantly altered by either AngII or PP2C $\alpha$ siRNA, indicating that the increased LC3 conversion observed with PP2C $\alpha$ knockdown 


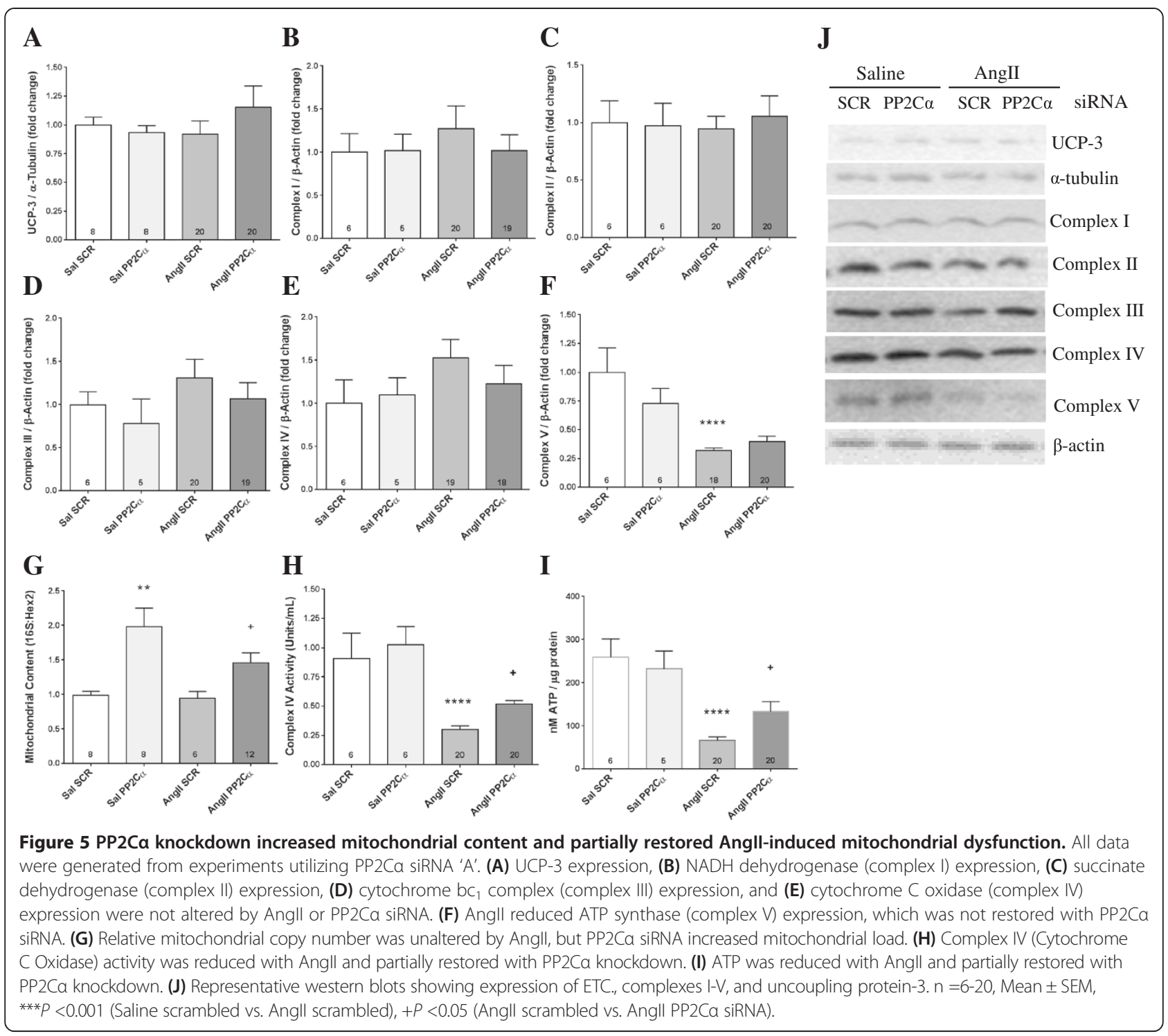

(Figure 7C) was not likely mediated by increased transcription of LC3.

Western blot data for p62 were verified by immunofluorescence on Gas cross sections showing an increase in p62-positive punctae with AngII and a reduction compared to AngII alone with knockdown of PP2C $\alpha$ (Figure 7J). Further, PP2C $\alpha$ siRNA increased the number LC3A-positive punctae compared to AngII alone (Figure $7 \mathrm{~K}$ ), indicating a greater number of autophagosomes with knockdown of PP2C $\alpha$ in AngII-infused Gas. PP2C $\alpha$ siRNA had no obvious effect on the number of p62 or LC3A-positive punctae in saline infused controls (Figure 7J and K). PP2C $\alpha$ siRNA also increased expression of beclin- 1 by 2.8 -fold $(P<0.05$, Figure $7 \mathrm{~A}$ and $\mathrm{D})$, further supporting increased autophagy with $\mathrm{PP} 2 \mathrm{C} \alpha$ knockdown, while AngII reduced expression of the lysosome marker lysosomal-associated membrane protein 1 (LAMP1) indicating that there may be a reduction in the number of lysosomes with AngII, but this reduction was not prevented by PP2C $\alpha$ siRNA (Figure 7A and E).

Given the observed changes in autophagy markers, ULK1 phosphorylation status was ascertained to obtain potential insights into mechanisms. ULK1 is an upstream mediator of autophagosome formation that is critical for autophagy, and its activity is known to be regulated by AMPK [36-39]. AngII reduced activating S555 phosphorylation of ULK1 by $39 \%(P<0.05)$, and this effect was completely reversed by PP2C $\alpha$ siRNA $(P<0.05$, Figure $7 F)$. Inhibitory $\mathrm{S} 757$ phosphorylation of ULK1 was increased by $86 \%$ with AngII $(P<0.01)$, and this increase was also almost completely reversed by knockdown of $\mathrm{PP} 2 \mathrm{C} \alpha$ $(P<0.05$, Figure $7 \mathrm{G})$. 


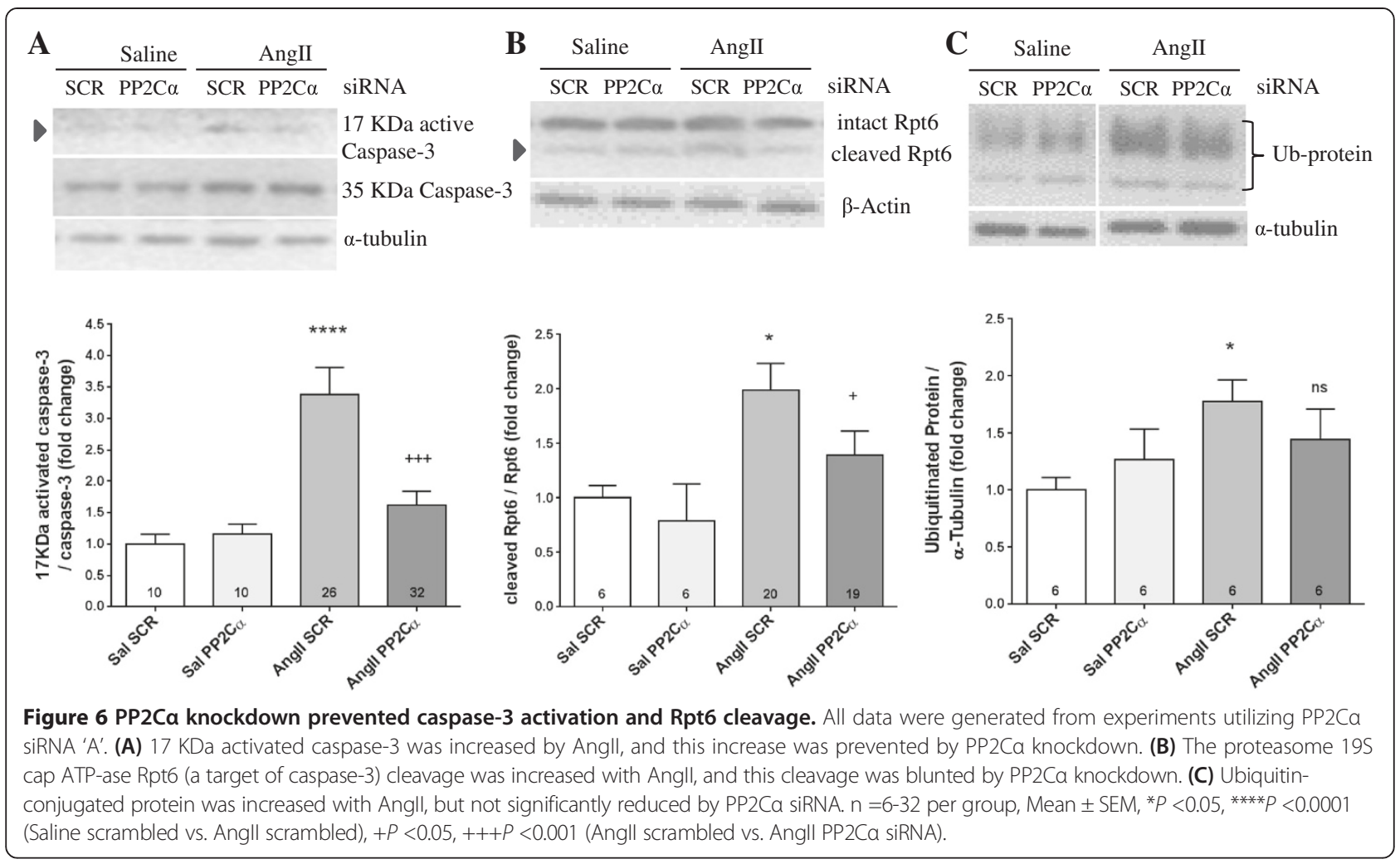

\section{Angll reduced expression of markers of mitochondrial fusion and fission via AMPK and MARCH5-indepentdent mechanisms}

Mitofusin-2 and Optic Atrophy 1 (OPA1) facilitate fusion of the outer and inner mitochondrial membranes, respectively. Mitochondrial fission is mediated by mitochondrial fission 1 protein (Fis1), mitochondrial fission factor (Mff), and dynamin-1-like protein (DRP1). Interestingly, Mitofusin-2, OPA1, Fis1, and DRP1 expression were all reduced by Ang II, indicating that Ang II inhibits both mitochondrial fusion and fission. AngII markedly reduced expression of mitofusin-2 (83\% decrease, $P<0.0001$ ), although PP2C $\alpha$ siRNA did not blunt this decrease (Figure 8A and B). Similarly, AngII also reduced expression of OPA1 by $68 \%(P<0.01)$ and knockdown of $\mathrm{PP} 2 \mathrm{C} \alpha$ did not prevent this reduction (Figure $8 \mathrm{~A}$ and $\mathrm{C}$ ). AngII reduced expression of Fis1 by $33 \%(P<0.05$, Figure $8 \mathrm{~A}$ and $\mathrm{D})$ and of DRP1 by $57 \%$ $(P<0.05$, Figure $8 \mathrm{~A}$ and $\mathrm{F})$, while $\mathrm{PP} 2 \mathrm{C} \alpha$ knockdown did not prevent these reductions (Figure 8A,D,F). PP2C $\alpha$ knockdown in saline-infused controls had no effect on expression of mitofusin-2, OPA1, DRP1, or Fis1 (Figure 8A$\mathrm{D}, \mathrm{F})$. Neither AngII nor PP2C $\alpha$ knockdown had any effect on expression of Mff (Figure 8G). DRP1 facilitates mitochondrial fission both via changes in expression and phosphorylation status [40], and while PP2C $\alpha$ siRNA had no effect on total DRP1 expression, it significantly increased activating DRP1 phosphorylation in AngII-infused Gas
(Figure 8A and E). Expression of membrane-associated RING-CH5 (MARCH5), an E3 ubiquitin ligase known to regulate both mitofusin-2 and Fis1 [41,42], was significantly reduced by AngII (Figure $8 \mathrm{~A}$ and $\mathrm{H}$ ), indicating that some other mechanism must be responsible for the reductions in mitofusin-2 and Fis1 with AngII. MARCH5 expression was not significantly altered by PP2C $\alpha$ knockdown in saline or AngII-infused groups (Figure 8A and $\mathrm{H}$ ).

\section{Discussion}

Our results indicate that $\mathrm{PP} 2 \mathrm{C} \alpha$ is a critical mediator of AngII wasting. Knockdown of PP2C $\alpha$ via electroporation of siRNA into Gas partially prevented AngII wasting, which is consistent with activation of AMPK being protective against wasting caused by AngII, as we have previously demonstrated [14]. Although there are technical limitations of siRNA electroporation into the skeletal muscle (that is, electroporation efficiency and siRNA effectiveness on PP2Ca mRNA), our PP2Ca knockdown significantly prevented Ang II wasting in vivo. Still, some components of AngII-wasting are PP2C $\alpha / A M P K-i n d e-$ pendent, or at least indirectly related, which may explain the partial rescue. We also demonstrate that the metabolic effects of AngII are critical, as PP2C $\alpha$ knockdown did not significantly alter the effects of AngII on Akt, FOXO, or expression of E3 ubiquitin ligases atrogin-1 or MURF1. This indicates that something other than the Akt-FoxO-E3 axis is predominately responsible for the 


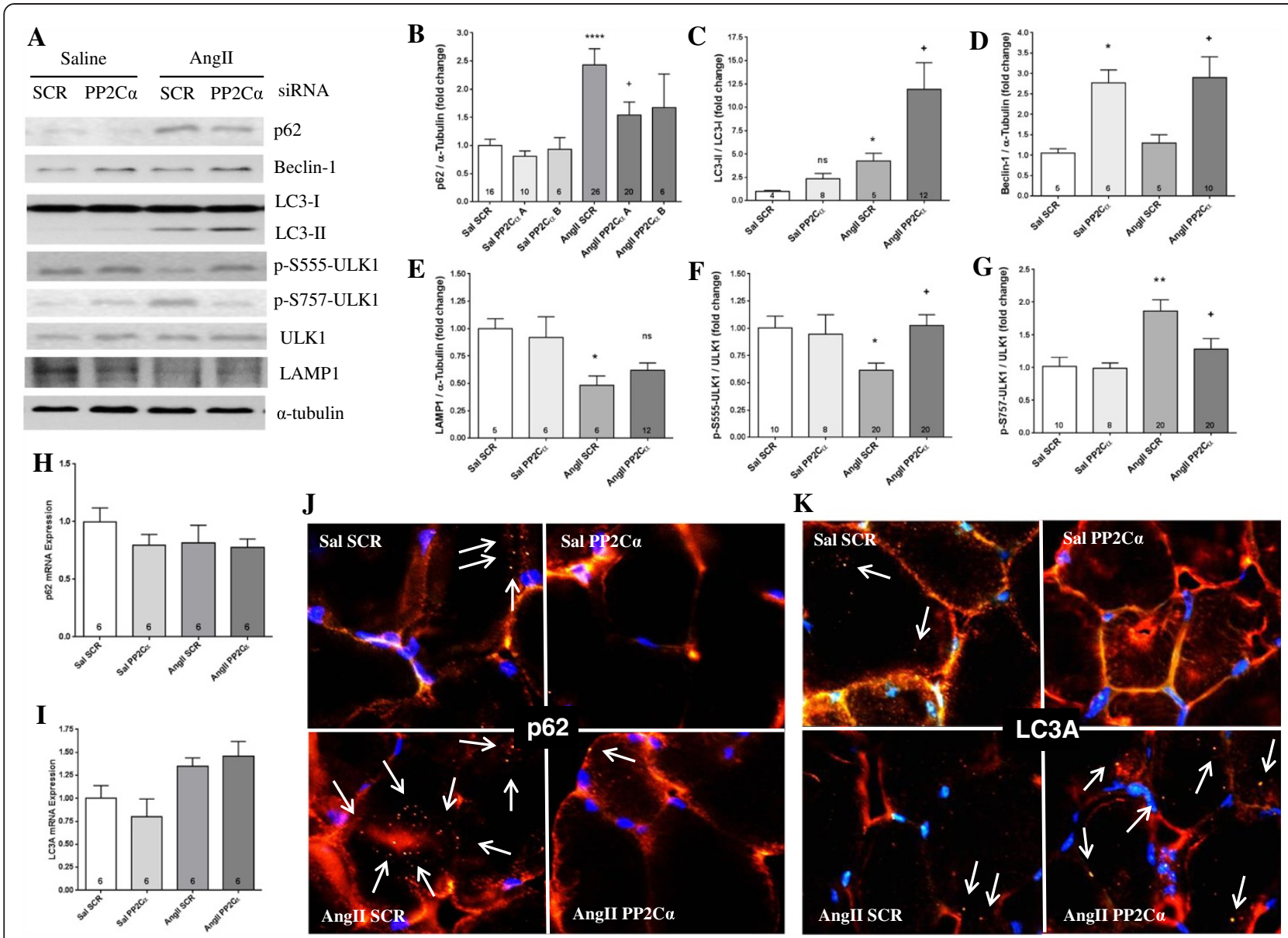

Figure 7 Angll inhibited autophagy while PP2Ca knockdown activated it via ULK1. (A) Representative western blots showing effects of Angll and PP2Ca siRNA on markers of autophagy. (B) Angll increased p62 accumulation (indicative of an inhibition of autophagy) and knockdown of PP2Ca prevented p62 accumulation. Specificity of the rescue was verified via utilization of two PP2Ca siRNAs, as indicated. All other data were generated from experiments utilizing PP2Ca siRNA 'A'. (C) LC3-II conversion was increased with Angll, but increased further by PP2Ca knockdown. (D) PP2Ca siRNA increased expression of the autophagy marker Beclin-1. (E) Angll reduced expression of the lysosome marker LAMP1, but this reduction was not restored by PP2Ca siRNA. (F) Angll decreased activating ULK1 phosphorylation, which was prevented by PP2Ca siRNA. (G) Angll increased inhibitory ULK1 phosphorylation, which was prevented by PP2Ca siRNA. (H, I) There was no transcriptional regulation of p62 (H), or LC3A (I) with either Angll or PP2Ca siRNA. (J) Representative immunohistochemical images showing the Angll-mediated increase in p62-positive punctae, and the prevention of that increase via knockdown of PP2Ca, showing inhibition of autophagy with Angll and restoration of autophagic flux with PP2Ca knockdown. (K) Representative immunohistochemical images showing PP2Ca siRNA increased autophagosome number as determined by LC3-positive punctae. $n=4-20$, Mean \pm SEM, ${ }^{*} P<0.05,{ }^{* *} P<0.01,{ }^{* * * *} P<0.0001$ (Saline scrambled vs. Angll scrambled), $+P<0.05$ (Angll scrambled vs. Angll PP2Ca siRNA).

rescue effects of PP2C $\alpha$ knockdown with respect to mitochondrial function, ATP, and muscle wasting. Finally, we have uncovered novel mechanisms linking elevated AngII to mitochondrial dysfunction in skeletal muscle. These mechanisms are both AMPK-dependent (reduced mitochondrial biogenesis via reduction in PGC- $1 \alpha /$ TFAM, and inhibition of mitophagy via inhibition of ULK1), and AMPK-independent (reductions in expression of important regulators of mitochondrial network dynamics).

Certainly, the UPP is very important for AngII wasting, and we have previously shown that AngII-mediated effects on Akt/FOXO phosphorylation and upregulation of atrogin-1 and MURF1 mRNA was prevented by direct
AMPK activation with AICAR [14]. It is important to point out that the experimental model in the present study may have some differences from our previously published work in that injection and electroporation of Gas muscles represents significant injury to the muscle, and may have induced the muscle's normal regenerative responses independent of AngII and/or PP2C $\alpha$ knockdown. Even so, while knockdown of PP2Ca did not significantly rescue from AngII-induced upregulations of atrogin-1, MURF1, and ubiquitin-conjugated proteins, there was a trend for a reduction in each of these endpoints, although these trends remain independent of the Akt-FOXO signaling axis. Further studies will be required to more closely 


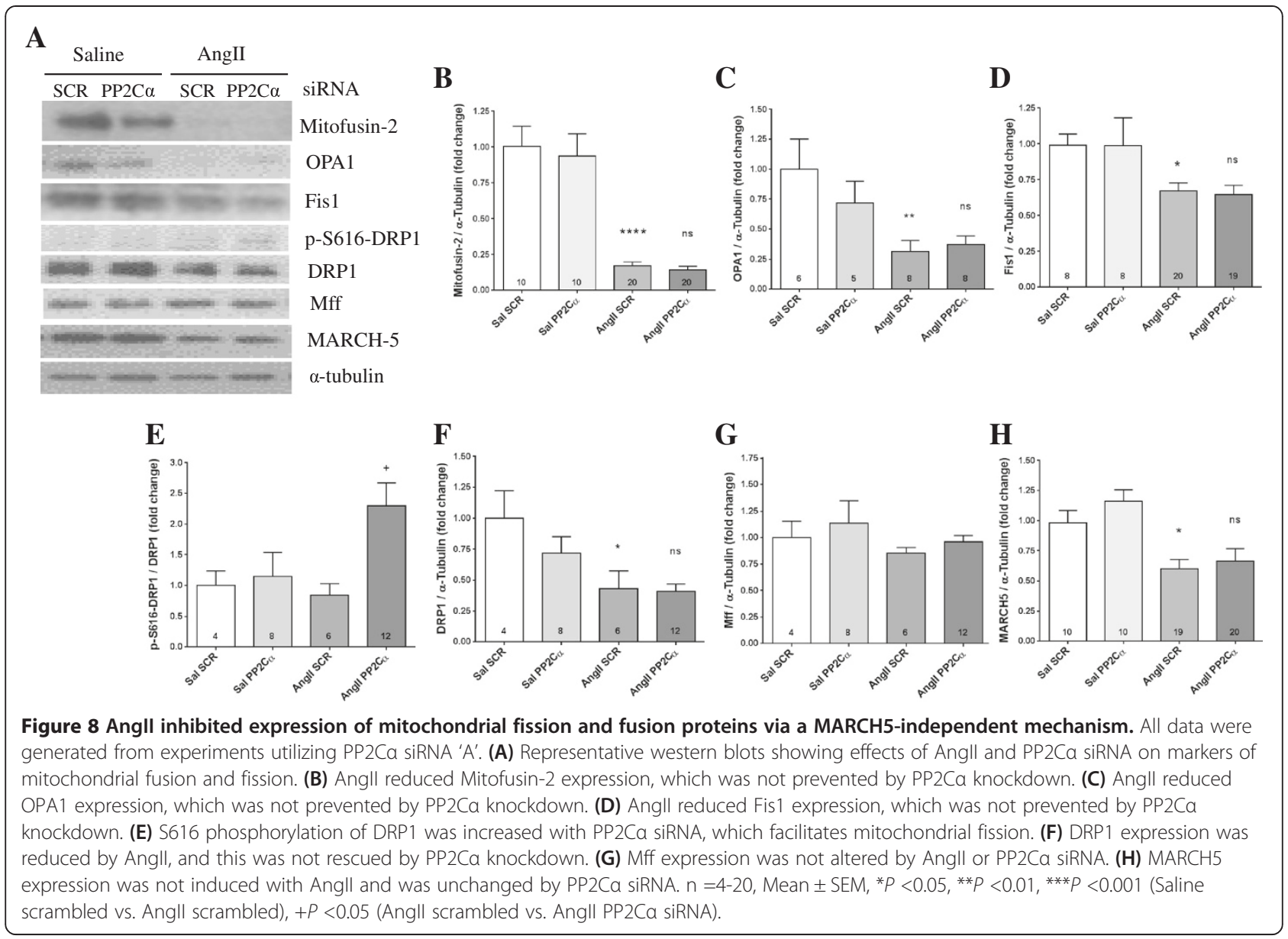

characterize the role of AngII-mediated AMPK inhibition on the UPP, but we have clearly shown that many of the effects of AngII on mitochondrial dysfunction are dependent on AMPK.

As AMPK is activated, PGC- $1 \alpha$ expression increases, in turn activating a program of mitochondrial biogenesis [31,32]. As such, AngII's effect to suppress AMPK phosphorylation and expression of PGC-1 $\alpha / \mathrm{NRF} 1 / \mathrm{TFAM}$ in skeletal muscle impairs generation of new mitochondria. The deleterious effects of AngII on mitochondrial biogenesis are reflected by AngII's marked suppression of mitochondrial ATP synthase (complex V) expression, complex IV activity, and ATP generation in Gas. Conversely, the ability of PP2C $\alpha$ knockdown to increase PGC-1 $\alpha$, NRF1, and TFAM expression acts in opposition to AngII by facilitating mitochondrial biogenesis and $\mathrm{PP} 2 \mathrm{C} \alpha$ siRNA partially restored mitochondrial activity and ATP toward basal levels, while significantly increasing mitochondrial copy number.

Healthy mitochondrial function is normally maintained both by generation of new mitochondria via PGC- $1 \alpha-$ NRF1-TFAM-mediated biogenesis, and by sequestration of damaged or defective mitochondria to autophagosomes and their subsequent degradation following fusion to lysosomes in a process known as mitophagy $[43,44]$. The specificity of mitophagy is mediated via mitochondrial fission and fusion, as well as by several mitophagy proteins, including p62, which binds ubiquitinated mitochondrial proteins and LC3 on the autophagosome to facilitate selective degradation of damaged mitochondria [44,45]. Because p62 is normally degraded along with the rest of the components of the autophagosome, its accumulation in the absence of transcriptional regulation indicates an inhibition of autophagy [44].

AngII significantly increased p62 protein without altering mRNA levels, indicating impairment of autophagy. While p62 clearance/accumulation is a reasonable index of autophagic flux in the absence of transcriptional regulation, it will be informative to measure flux directly in future studies, for instance by tracking LC3 turnover alongside p62 accumulation [46]. The partial restoration of p62 to basal levels, along with increased expression of Beclin-1 and conversion of LC3-II with PP2Ca knockdown, indicates that AngII decreased autophagy via 
inhibition of AMPK. LC3-II conversion is an important early step in autophagosome formation and a higher ratio of LC3-II to LC3-I indicates a greater number of autophagosomes and usually indicates an induction of autophagy, although increased LC3 expression and conversion can also indicate an inhibition of downstream autophagic processes [47]. Because AngII appeared to reduce the expression of the lysosome marker LAMP1, the increased LC3-II conversion with AngII compared to baseline may implicate such a downstream inhibition in addition to the upstream effects on ULK1. Given that AngII increased p62 accumulation while leaving beclin-1 expression unchanged, the AngII-mediated increase in LC3-II conversion over control likely does not indicate an activation of autophagy over basal rates. Indeed, while it did not reach statistical significance, there appears to be a modest trend for increased LC3A gene expression with AngII, which may also explain the increase in LC3II conversion over basal conditions with AngII. Knockdown of PP2Ca in the presence of AngII further increased LC3-II conversion, but reduced p62 and also increased beclin-1, indicating that AngII-mediated inhibition of AMPK inhibited autophagy.

Failure of the cell to clear damaged mitochondrial proteins results in the accumulation of dysfunctional mitochondria and a reduced ability to meet cellular energetic demands. This deficit is accentuated in our model (where AMPK activation is impaired by AngII) because AMPK activation is the normal physiologic response to decreased ATP availability. Although AMPK activation can be associated with mitochondrial dependent muscle wasting, and inhibition of AMPK has been shown to restore muscle size in myofibers with altered mitochondria [45], the opposite appears to be the case with regards to wasting caused by AngII [14].

In the current study, p62 accumulation with AngII indicates impairment in the rate of autophagy. This is corroborated by the accumulation of dysfunctional complex IV in AngII-infused muscle, as demonstrated by the marked reduction in complex IV activity, even while total complex IV expression tended to increase. The accumulation of dysfunctional mitochondria is reflected in the reduction of ATP in response to AngII while mitochondrial load remained unchanged. Activation of AMPK signaling via knockdown of PP2C $\alpha$ acted in opposition to AngII by facilitating autophagy as evidenced by reduced p62 accumulation, and improved mitochondrial function as indicated by increased complex IV activity, and increased ATP. Further, the degree to which PP2C $\alpha$ siRNA activated mitochondrial biogenesis outweighed the degree to which mitophagy was activated since total mitochondrial content was increased by knockdown of PP2C $\alpha$. As such, the net effect of PP2C $\alpha$ knockdown during infusion of AngII is a greater number of total mitochondria and a simultaneously higher proportion of fully functional, more efficient mitochondria, leading to increased energy production and protection from AngII wasting.

ULK1 is a critical mediator of autophagy [36-39], acting at the most upstream stages of nucleation and initial autophagosome formation. The ULK1 complex is differentially activated and inhibited by AMPK and mTORC1, respectively [36-39,44,47]. AngII reduced activating ULK1 phosphorylation, while simultaneously inducing inhibitory ULK1 phosphorylation in Gas, effects which are likely mediated, at least in part, by AngII's inhibitory effects on AMPK in muscle, and which serve to inhibit autophagy in muscle. Activation of AMPK via knockdown of its phosphatase PP2C $\alpha$ alleviated the inhibitory effect of AngII on AMPK and restored ULK1 phosphorylation status to basal levels, in turn restoring autophagic flux to normal levels. The ability of PP2Ca knockdown to oppose the inhibitory effects of AngII on ULK1 activation is consistent with a mechanism whereby AngII impairs autophagy and AMPK acts in opposition to activate it in skeletal muscle.

Parallel to its inhibitory effects on the PGC- $1 \alpha-N R F 1-$ TFAM axis (mitochondrial biogenesis), ULK1 (autophagosome formation), and p62 clearance (autophagic flux), AngII also significantly reduced expression of several markers of mitochondrial fusion (mitofusin-2, OPA1) and fission (Fis1, DRP1), suggesting that AngII also acts to inhibit both mitochondrial fusion and mitochondrial fission. Mitofusin-2 is located on the outer mitochondrial membrane, while OPA1 is localized to the inner mitochondrial membrane and both are critical for the initiation of mitochondrial fusion $[41,44,48]$. Fusion of mitochondria into complex networks helps to increase overall mitochondrial efficiency by compensating for damaged or dysfunctional oxidative phosphorylation machinery and tends to be induced during times of high energetic demand [44]. Therefore, inhibition of mitochondrial fusion by AngII would prevent the muscle from compensating for the deleterious effects of AngII on energy balance, and could play a role in ATP depletion with AngII. Fis1 and Mff are localized to the outer mitochondrial membrane, while DRP1 associates with these proteins to form a complex which facilitates fission of the mitochondrial network [40-42,48]. Both DRP1 expression and activity are important for mitochondrial fission, and its activity is modulated via phosphorylation by several upstream kinases including Cdk1/cyclin B, and protein kinase A [49]. Mitochondrial fission is important because it allows the cell to group damaged mitochondrial components together so that they can be selectively recycled via mitophagy (thereby leaving functioning mitochondria intact). The ability of AngII to reduce Fis1 and DRP1 expression may further facilitate AngII-impairment of selective mitophagy and could also help explain the build-up of damaged mitochondrial proteins and energy depletion in response to AngII, while increased activating 


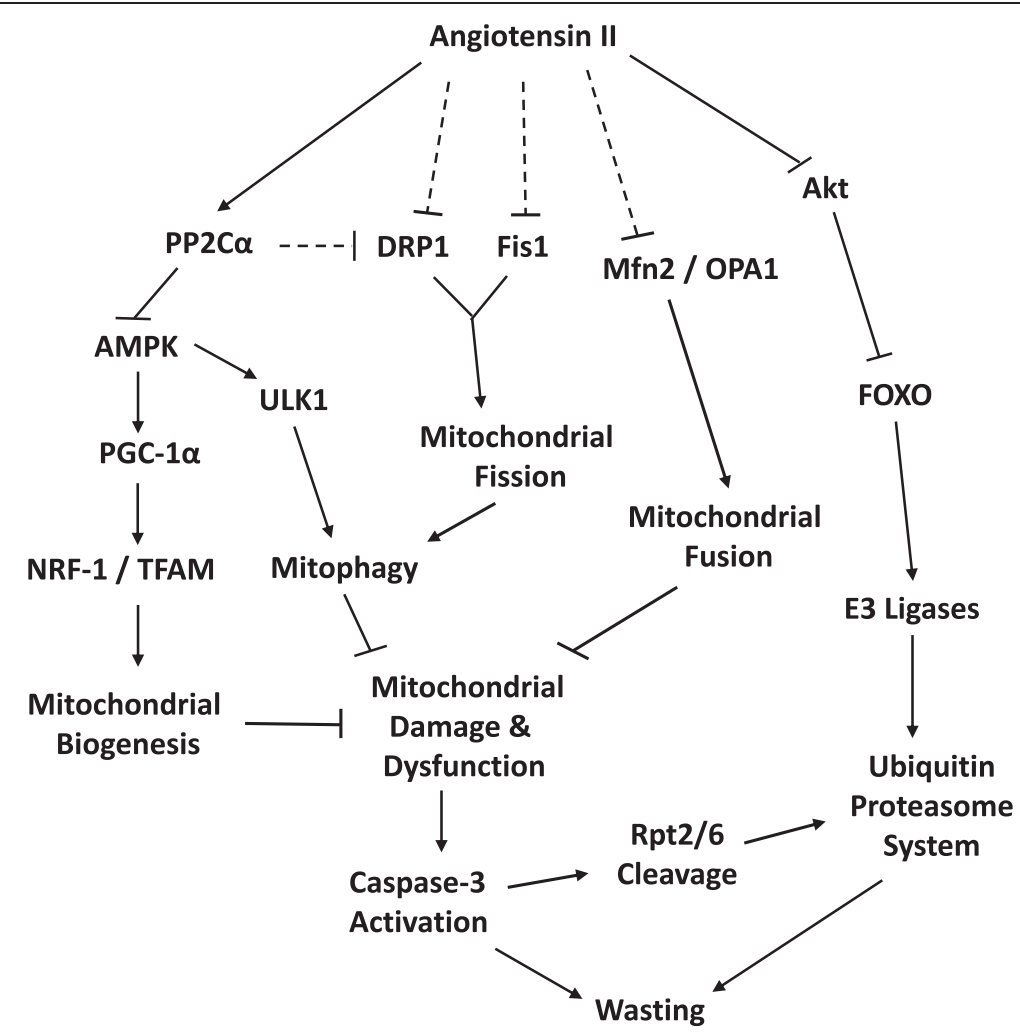

Figure 9 Proposed model whereby Angll infusion leads to mitochondrial dysfunction and skeletal muscle wasting. Parallel to the well-characterized Angll-mediated activation of the FOXO-E3-UPS axis via inhibition of Akt, Angll also induces expression of the phosphatase PP2Ca, which dephosphorylates and inactivates AMPK. This leads to reduced PGC-1a, NRF1, and TFAM expression (less mitochondrial biogenesis), and reduced ULK1 activity. The Angll-mediated reduction in ULK1 activation inhibits a critical early step in the autophagy pathway and prevents recycling of damaged mitochondria (mitophagy). Angll also inhibits both mitochondrial fission and fusion through predominately AMPK-independent pathways, which likely contribute to mitochondrial dysfunction caused by elevated Angll. Prolonged mitochondrial dysfunction and energy depletion ultimately leads to release of caspase-3, initiation of apoptosis, and wasting.

DRP1 phosphorylation with PP2C $\alpha$ siRNA may act in opposition to facilitate fission and selective mitophagy.

MARCH5 is an E3 ligase that regulates the expression of both mitofusin-2 and Fis1 via UPP-mediated degradation of these proteins [41]. Its expression is not significantly increased with AngII. In fact, MARCH5 expression was significantly reduced by AngII, making it unlikely that MARCH5 mediates the AngII-induced reductions in mitofusin-2 and Fis1. Further, knockdown of PP2C $\alpha$ reversed the effects of AngII on PGC- $1 \alpha$ and autophagy, but did not reverse the effects of AngII on mitofusin-2, OPA1, DRP1, or Fis1 expression, suggesting that AMPK is not directly involved in mediating the expression of these proteins, and that the ability of PP2C $\alpha$ knockdown to improve mitochondrial recycling is primarily through restoration of ULK1 activity and autophagosome formation rather than via alterations in mitochondrial fission/fusion. Still, the overall relationship between AngII, AMPK, and the dynamic remodeling of the mitochondrial network will require further studies, since PGC-1 $\alpha[50,51]$ and FOXO3a [44] have been shown to play a role in mitochondrial fusion and fission, respectively, and both are known to be regulated by AMPK.

\section{Conclusions}

Our data show that AngII-mediated skeletal muscle wasting is characterized by impairment of mitochondrial biogenesis, fission, fusion, and autophagy, thereby preventing normal mitochondrial recycling and leading to mitochondrial dysfunction, ATP depletion, and wasting (summarized in Figure 9). Further, we have shown that AngII wasting and its effects on mitochondrial function are mediated at least in part via upregulation of PP2C $\alpha$ in muscle. Thus, our studies elucidate novel mechanisms underlying AngII wasting and suggest a therapeutic potential for activators of AMPK in wasting conditions where the reninangiotensin system is activated.

\section{Abbreviations}

AICAR: 5-aminoimidazole-4-carboxamide ribonucleoside; Akt: Protein kinase B; AMPK: AMP-Activated Protein Kinase; Angll: Angiotensin II; ANOVA: analysis of variance; ATP: Adenosine triphosphate; atrogin-1: Atrogin-1/MAFbx,

Fbxo32; CHF: Congestive heart failure; complex I: NADH dehydrogenase; 
complex II: succinate dehydrogenase; complex III: cytochrome bc complex; complex IV: Cytochrome C oxidase; complex V: ATP synthase;

DRP1: Dynamin-1-like protein; Fis1: Mitochondrial fission 1 protein;

FOXO: Forkhead box protein class O; Gas: Gastrocnemius muscle;

IGF-1: Insulin-like growth factor 1; LAMP1: Lysosomal-associated membrane protein 1; LC3: microtubule-associated protein 1 light chain 3;

MARCH5: Membrane-associated RING-CH5; Mff: Mitochondrial fission factor; mtDNA: Mitochondrial deoxyribonucleic acid; mTOR: Mammalian target of rapamycin; MuRF1: Muscle RING-finger protein-1, Trim63; NRF1: nNuclear respiratory factor 1; OCT: Optimal cutting temperature; OPA1: Optic atrophy 1; p62: p62/SQSTM1; PGC-1a: Peroxisome proliferator -activated receptor- $\gamma$ coactivator 1-a; PP2Ca: Protein phosphatase 2C-alpha; RT-PCR: Reverse transcriptase polymerase chain reaction; SEM: Standard error of the mean; siRNA: Small interfering ribonucleic acid; TFAM: Mitochondrial transcription factor A; UCP3: Uncoupling protein-3; ULK1: UNC-51-like kinase 1;

UPP: Ubiquitin proteasome pathway; WB: Western blot.

\section{Competing interests}

The authors declare that they have no competing interests.

\section{Authors' contributions}

AMT carried out the animal experiments, performed the molecular assays and immunoblots, participated in the design of the study, performed the statistical analysis, and drafted the manuscript. TY performed the quantification of muscle fiber cross sectional area, participated in the design of the study, and helped to revise manuscript. SS performed the immunohistochemical analysis of p62 and LC3 positive punctae in Gas cross sections and participated in the design of the study. PD conceived of the study and participated in its design, coordination, and interpretation of results, and helped to revise the manuscript. All authors read and approved the final manuscript.

\section{Acknowledgements}

This research was supported by National Institutes of Health Grants R01HL070241, R01HL080682, R21-HL113705-01A1 P30GM103337, U54GM104940 and P20GM103629, and American Heart Association Grant-in-Aid 13GRNT17230069.

Received: 5 June 2014 Accepted: 10 October 2014

Published: 30 October 2014

\section{References}

1. Anker SD, Negassa A, Coats AJ, Afzal R, Poole-Wilson PA, Cohn JN, Yusuf S: Prognostic importance of weight loss in chronic heart failure and the effect of treatment with angiotensin-converting-enzyme inhibitors: an observational study. Lancet 2003, 361:1077-1083.

2. Levenson JW, Skerrett PJ, Gaziano JM: Reducing the global burden of cardiovascular disease: the role of risk factors. Prev Cardiol 2002 5:188-199.

3. Tan BH, Fearon KC: Cachexia: prevalence and impact in medicine. Curr Opin Clin Nutr Metab Care 2008, 11:400-407.

4. Du J, Hu Z, Mitch WE: Cellular signals activating muscle proteolysis in chronic kidney disease: a two-stage process. Int J Biochem Cell Biol 2005, 37:2147-2155

5. Rajan V, Mitch WE: Ubiquitin, proteasomes and proteolytic mechanisms activated by kidney disease. Biochim Biophys Acta 2008, 1782:795-799.

6. Brink M, Price SR, Chrast J, Bailey JL, Anwar A, Mitch WE, Delafontaine P: Angiotensin II induces skeletal muscle wasting through enhanced protein degradation and down-regulates autocrine insulin-like growth factor I. Endocrinology 2001, 142:1489-1496.

7. Brink M, Wellen J, Delafontaine P: Angiotensin II causes weight loss and decreases circulating insulin-like growth factor I in rats through a pressor-independent mechanism. J Clin Invest 1996, 97:2509-2516.

8. Folli F, Kahn CR, Hansen H, Bouchie JL, Feener EP: Angiotensin II inhibits insulin signaling in aortic smooth muscle cells at multiple levels. A potential role for serine phosphorylation in insulin/angiotensin II crosstalk. J Clin Invest 1997, 100:2158-2169.

9. Folli F, Saad MJ, Velloso L, Hansen H, Carandente O, Feener EP, Kahn CR: Crosstalk between insulin and angiotensin II signalling systems. Exp Clin Endocrinol Diabetes 1999, 107:133-139.

10. Song YH, Li Y, Du J, Mitch WE, Rosenthal N, Delafontaine P: Muscle-specific expression of IGF-1 blocks angiotensin II-induced skeletal muscle wasting. J Clin Invest 2005, 115:451-458.
11. Workeneh BT, Mitch WE: Review of muscle wasting associated with chronic kidney disease. Am J Clin Nutr 2010, 91:1128S-1132S

12. Yoshida T, Semprun-Prieto L, Sukhanov S, Delafontaine P: IGF-1 prevents ANG II-induced skeletal muscle atrophy via Akt- and Foxo-dependent inhibition of the ubiquitin ligase atrogin-1 expression. Am J Physiol Heart Circ Physiol 2010, 298:H1565-H1570.

13. Zhang L, Du J, Hu Z, Han G, Delafontaine P, Garcia G, Mitch WE: IL-6 and serum amyloid A synergy mediates angiotensin II-induced muscle wasting. J Am Soc Nephrol 2009, 20:604-612.

14. Tabony AM, Yoshida T, Galvez S, Higashi Y, Sukhanov S, Chandrasekar B, Mitch WE, Delafontaine P: Angiotensin II upregulates protein phosphatase 2Calpha and inhibits AMP-activated protein kinase signaling and energy balance leading to skeletal muscle wasting. Hypertension 2011, 58:643-649.

15. Yoshida T, Semprun-Prieto L, Wainford RD, Sukhanov S, Kapusta DR, Delafontaine $P$ : Angiotensin II reduces food intake by altering orexigenic neuropeptide expression in the mouse hypothalamus. Endocrinology 2012, 153:1411-1420.

16. Yoshida T, Tabony AM, Galvez S, Mitch WE, Higashi Y, Sukhanov S, Delafontaine P: Molecular mechanisms and signaling pathways of angiotensin II-induced muscle wasting: potential therapeutic targets for cardiac cachexia. Int J Biochem Cell Biol 2013, 45:2322-2332.

17. Wang XH, Zhang L, Mitch WE, LeDoux JM, Hu J, Du J: Caspase-3 cleaves specific $19 \mathrm{~S}$ proteasome subunits in skeletal muscle stimulating proteasome activity. J Biol Chem 2010, 285:21249-21257.

18. Mitsuishi M, Miyashita K, Muraki A, Itoh H: Angiotensin II reduces mitochondrial content in skeletal muscle and affects glycemic control. Diabetes 2009, 58:710-717.

19. Sato H, Watanabe A, Tanaka T, Koitabashi N, Arai M, Kurabayashi M, Yokoyama T: Regulation of the human tumor necrosis factor-alpha promoter by angiotensin II and lipopolysaccharide in cardiac fibroblasts: different cis-acting promoter sequences and transcriptional factors. J Mol Cell Cardiol 2003, 35:1197-1205.

20. Sukhanov S, Semprun-Prieto L, Yoshida T, Michael Tabony A, Higashi Y, Galvez S, Delafontaine P: Angiotensin II, oxidative stress and skeletal muscle wasting. Am J Med Sci 2011, 342:143-147.

21. Touyz RM, Tabet F, Schiffrin EL: Redox-dependent signalling by angiotensin II and vascular remodelling in hypertension. Clin Exp Pharmacol Physiol 2003, 30:860-866.

22. Zera T, Ufnal M, Szczepanska-Sadowska E: Central TNF-alpha elevates blood pressure and sensitizes to central pressor action of angiotensin II in the infarcted rats. J Physiol Pharmacol 2008, 59(Suppl 8):117-121.

23. Yoshida T, Galvez S, Tiwari S, Rezk BM, Semprun-Prieto L, Higashi Y, Sukhanov S, Yablonka-Reuveni Z, Delafontaine P: Angiotensin II inhibits satellite cell proliferation and prevents skeletal muscle regeneration. J Biol Chem 2013, 288:23823-23832.

24. Broderick KE, Chan A, Lin F, Shen X, Kichaev G, Khan AS, Aubin J, Zimmermann TS, Sardesai NY: Optimized in vivo transfer of small interfering RNA targeting dermal tissue using in vivo surface electroporation. Mol Ther Nucleic Acids 2012, 1:e11.

25. Finocchietto P, Barreyro F, Holod S, Peralta J, Franco MC, Mendez C, Converso DP, Estevez A, Carreras MC, Poderoso Jj: Control of muscle mitochondria by insulin entails activation of Akt2-mtNOS pathway: implications for the metabolic syndrome. PLoS One 2008, 3:e1749.

26. Golzio M, Mazzolini L, Moller P, Rols MP, Teissie J: Inhibition of gene expression in mice muscle by in vivo electrically mediated siRNA delivery. Gene Ther 2005, 12:246-251.

27. Gonzalez-Villalobos RA, Seth DM, Satou R, Horton H, Ohashi N, Miyata K, Katsurada A, Tran DV, Kobori H, Navar LG: Intrarenal angiotensin II and angiotensinogen augmentation in chronic angiotensin II-infused mice. Am J Physiol Renal Physiol 2008, 295:F772-F779.

28. Graziani G, Badalamenti S, Del Bo A, Marabini M, Gazzano G, Como G, Vigano E, Ambroso G, Morganti A: Abnormal hemodynamics and elevated angiotensin II plasma levels in polydipsic patients on regular hemodialysis treatment. Kidney Int 1993, 44:107-114.

29. Masson S, Latini R, Bevilacqua M, Vago T, Sessa F, Torri M, Anesini A, Salio M, Pasotti E, Agnello D, Santoro L, Catania A, Ghezzi P, Moccetti T, Maggioni AP: Within-patient variability of hormone and cytokine concentrations in heart failure. Pharmacol Res 1998, 37:213-217.

30. Roig E, Perez-Villa F, Morales M, Jimenez W, Orus J, Heras M, Sanz G: Clinical implications of increased plasma angiotensin II despite ACE inhibitor therapy in patients with congestive heart failure. Eur Heart $J$ 2000, 21:53-57. 
31. Irrcher I, Ljubicic V, Hood DA: Interactions between ROS and AMP kinase activity in the regulation of PGC-1alpha transcription in skeletal muscle cells. Am J Physiol Cell Physiol 2009, 296:C116-C123.

32. McGee SL, Hargreaves M: AMPK-mediated regulation of transcription in skeletal muscle. Clin Sci (Lond) 2010, 118:507-518.

33. Pejznochova M, Tesarova M, Hansikova H, Magner M, Honzik T, Vinsova $K$, Hajkova Z, Havlickova V, Zeman J: Mitochondrial DNA content and expression of genes involved in mtDNA transcription, regulation and maintenance during human fetal development. Mitochondrion 2010, 10:321-329.

34. Kubli DA, Gustafsson AB: Mitochondria and mitophagy: the yin and yang of cell death control. Circ Res 2012, 111:1208-1221.

35. Marzetti E, Calvani R, Cesari M, Buford TW, Lorenzi M, Behnke BJ, Leeuwenburgh $C$ : Mitochondrial dysfunction and sarcopenia of aging: from signaling pathways to clinical trials. Int J Biochem Cell Biol 2013, 45:2288-2301.

36. Lee JW, Park S, Takahashi Y, Wang HG: The association of AMPK with ULK1 regulates autophagy. PLoS One 2010, 5:e15394.

37. Mizushima N, Noda T, Yoshimori T, Tanaka Y, Ishii T, George MD, Klionsky DJ, Ohsumi M, Ohsumi Y: A protein conjugation system essential for autophagy. Nature 1998, 395:395-398.

38. Mizushima N, Sugita H, Yoshimori T, Ohsumi Y: A new protein conjugation system in human. The counterpart of the yeast Apg12p conjugation system essential for autophagy. J Biol Chem 1998, 273:33889-33892.

39. Sanchez AM, Csibi A, Raibon A, Cornille K, Gay S, Bernardi H, Candau R: AMPK promotes skeletal muscle autophagy through activation of forkhead FoxO3a and interaction with Ulk1. J Cell Biochem 2012, 113:695-710.

40. MacVicar TD, Lane JD: Impaired OMA1-dependent cleavage of OPA1 and reduced DRP1 fission activity combine to prevent mitophagy in cells that are dependent on oxidative phosphorylation. J Cell Sci 2014, 127:2313-2325.

41. Park YY, Lee S, Karbowski M, Neutzner A, Youle RJ, Cho H: Loss of MARCH5 mitochondrial E3 ubiquitin ligase induces cellular senescence through dynamin-related protein 1 and mitofusin 1. J Cell Sci 2010, 123:619-626.

42. Xu S, Pi H, Chen Y, Zhang N, Guo P, Lu Y, He M, Xie J, Zhong M, Zhang Y, Yu Z, Zhou Z: Cadmium induced Drp1-dependent mitochondrial fragmentation by disturbing calcium homeostasis in its hepatotoxicity. Cell Death Dis 2013, 4:e540.

43. Hulmi JJ, Oliveira BM, Silvennoinen M, Hoogaars WM, Pasternack A, Kainulainen $\mathrm{H}$, Ritvos $\mathrm{O}$ : Exercise restores decreased physical activity levels and increases markers of autophagy and oxidative capacity in myostatin/activin-blocked mdx mice. Am J Physiol Endocrinol Metab 2013, 305:E171-E182.

44. Romanello V, Sandri M: Mitochondrial biogenesis and fragmentation as regulators of protein degradation in striated muscles. J Mol Cell Cardiol 2013, 55:64-72.

45. Romanello V, Guadagnin E, Gomes L, Roder I, Sandri C, Petersen Y, Milan G, Masiero E, Del Piccolo P, Foretz M, Scorrano L, Rudolf R, Sandri M: Mitochondrial fission and remodelling contributes to muscle atrophy. EMBO J 2010, 29:1774-1785.

46. Zhang XJ, Chen S, Huang KX, Le WD: Why should autophagic flux be assessed? Acta Pharmacol Sin 2013, 34:595-599.

47. Mizushima N, Yoshimori T, Levine B: Methods in mammalian autophagy research. Cell 2010, 140:313-326.

48. van der Bliek AM, Shen Q, Kawajiri S: Mechanisms of mitochondrial fission and fusion. Cold Spring Harb Perspect Biol 2013, 5: doi: 10.1101/cshperspect. a011072.

49. Knott AB, Perkins G, Schwarzenbacher R, Bossy-Wetzel E: Mitochondrial fragmentation in neurodegeneration. Nat Rev Neurosci 2008, 9:505-518.

50. Rowe GC, Jiang A, Arany Z: PGC-1 coactivators in cardiac development and disease. Circ Res 2010, 107:825-838.

51. Wenz T: PGC-1alpha activation as a therapeutic approach in mitochondrial disease. IUBMB Life 2009, 61:1051-1062.

doi:10.1186/2044-5040-4-20

Cite this article as: Tabony et al:: Protein phosphatase $2 \mathrm{C}$-alpha knockdown reduces angiotensin II-mediated skeletal muscle wasting via restoration of mitochondrial recycling and function. Skeletal Muscle 2014 4:20.

\section{Submit your next manuscript to BioMed Central and take full advantage of:}

- Convenient online submission

- Thorough peer review

- No space constraints or color figure charges

- Immediate publication on acceptance

- Inclusion in PubMed, CAS, Scopus and Google Scholar

- Research which is freely available for redistribution 